\title{
21. CHARACTERIZATION OF THE ORGANIC MATTER IN A SITE 147 CORE FROM THE CARIACO TRENCH
}

\author{
Thomas C. Hoering, Geophysical Laboratory, Carnegie Institution of Washington, Washington, D.C.
}

\section{INITIAL TREATMENT}

A section near the middle of core 10 from Site 147B collected at an approximate depth of 80 meters below the sediment surface, was freeze dried. A weight loss of 35.2 percent occurred. A 500 gram sample of the dried sediment was extracted twice with benzene-methanol mixture in an ultrasonic extractor. The extracts were combined and evaporated in a rotating, vacuum evaporator. They accounted for 0.10 weight percent of the starting, dried sediment. The residue was then extracted with dilute sodium hydroxide and a humic acid fraction isolated and purified. It represented only 0.0034 percent. The residue was then neutralized, freeze-dried and split into two samples. The first was pyrolyzed overnight in a flowing stream of hydrogen at a pressure of one atmosphere and a temperature of $350^{\circ} \mathrm{C}$. The pyrolysate was collected in a trap chilled with dry ice. The residue in the pyrolysis tube was extracted with benzene-methanol and combined with the material in the trap. The weight of the benzene-soluble material after pyrolysis was 0.10 percent of the dried sediment. The second half of the extracted sediment was suspended in two liters of six normal sulfuric acid. Excess potassium dichromate was added and 500 milliliters of distillate was steam distilled from the refluxing reaction mixture. The distillate and the residue after oxidation were extracted separately with diethyl ether in a liquid-liquid extractor to obtain volatile and non-volatile acid fractions.

\section{BENZENE-METHANOL EXTRACT}

A survey of the distribution of carbon skeletons and types of oxygenated functional groups present in the benzene-methanol extract was made by the following technique. All oxygenated functional groups were reduced to deuterated alcohols by lithium aluminum deuteride. The alcohols were converted to iodides and then further reduced to deuterated hydrocarbons by lithium aluminum deuteride. The steps are outlined using a carboxylic acid as an example.

$$
\begin{aligned}
& \mathrm{RCOOH}+\mathrm{LiAlD}_{4} \rightleftharpoons \mathrm{RCD}_{2} \mathrm{OH} \\
& \mathrm{RCD}_{2} \mathrm{OH}+\mathrm{HI} \rightleftharpoons \mathrm{RCD}_{2} \mathrm{I}+\mathrm{H}_{2} \mathrm{O} \\
& \mathrm{RCD}_{2} \mathrm{I}+\mathrm{LiAlD}_{4} \rightleftharpoons \mathrm{RCD}_{3}
\end{aligned}
$$

Carbon skeletons are not rearranged during the reactions. The amount and position of deuterium labelling indicates the types of functional groups that were reduced. Preexisting hydrocarbons will not have deuterium labelling; fatty alcohols will give hydrocarbons with one D atom; fatty acids will give hydrocarbons with three $\mathrm{D}$ atoms.
After the reduction steps, saturated hydrocarbons were isolated by silica gel chromotography. A small amount of C-14 tagged palmitic acid was added to the extract and carried through the reduction procedure. The yield of the tracer as hexadecane was 43.3 percent. When corrected for losses, the reduced deuterated hydrocarbons accounted for 24.2 percent of the benzene-methanol extract. A gas chromatogram of the hydrocarbons is shown in Figure 1.

The hydrocarbons were separated by urea and thiourea adductination and individual components isolated by preparative gas-liquid chromotography. They were introduced into a CEC-491 mass spectrometer through a gas chromatograph with a helium separator. The relative abundance of the deuterated parent peaks in the mass spectra were measured to determine the amount of deuterium labelling.

The reduced, deuterated normal hydrocarbons were predominately of an even number of carbon atoms in the range of from $\mathrm{C}_{12}$ to $\mathrm{C}_{32}$. The ratio of normal hydrocarbons with an even number of carbons to those with an odd number was about eight to one. The even carbon numbered alkanes were approximately an equimolar mixture of monodeuterated and trideuterated molecules. The odd carbon numbered alkanes with greater than twenty-five carbons were about ten percent unlabelled species; the remainder having one and three deuterium atoms. The branched chained hydrocarbons were exclusively monodeuterated, indicating that they arose from an alcohol precursor.

This experiment shows that fatty alcohols and acids are in great excess over saturated paraffins with comparable carbon skeletons. The comparable abundance of alcohols and acids is surprising. In the few literature reports on fatty alcohols in sediments, it has been found that acids greatly outnumber alcohols. A study of this phenomena down the length of the Cariaco Trench Core should indicate if this is due to reactions occurring during the early stages of diagenesis of the organic matter in marine sediments.

\section{PYROLYSIS}

The benzene-soluble pyrolysate was fractionated by silica gel chromotography. Saturated hydrocarbons and olefins were eluted by hexane and accounted for 28 percent of the pyrolysate. Subsequent elution of the column with more polar solvents removed a red band. After purification, it gave an adsorbtion spectrum of metallo-porphyrins. A gas chromatogram of the total hexane eluate is shown in Figure 2 with the identification of some of the major peaks. The urea adduct of the hexane eluate produced a smooth distribution of normal paraffins and olefins that reached a maximum at $\mathrm{C}_{22} \mathrm{H}_{46}$ with no odd carbon preference. The normal olefins were about 15 percent of the normal paraffins. 


\section{NON-VOLATILE ACIDS AFTER OXIDATION OF THE RESIDUE}

The ether extract of the residue after oxidation with chromic acid was esterified with methanol-boron trifluoride. The esters accounted for 0.044 percent of the starting sediment. Gas chromotography and mass spectrometry was used to identify major components. Dicarboxylic acids from succinic through pimelic and keto- or hydroxy-acids of similar carbon numbers were in the majority. A surprising result was the low yield of fatty acids with greater than 12 carbon atoms. Other recent sediments examined in this laboratory by this technique gave easily detectable amounts. Although chromic acid oxidation is a fairly drastic degradation procedure, fatty acids are only slowly oxidized and survive.

\section{STEAM VOLATILE ACIDS AFTER OXIDATION OF THE RESIDUE}

Volatile acids accounted for about 0.3 percent of the starting sediment. Gas chromotography and mass spectrometry of both the free acids and their butyl esters showed that normal, aliphatic acids from acetic through decanoic were the major components. Acetic, propionic and butyric acids were preponderant. Minor amounts of branched chain aliphatic acids and benzoic acid were identified. These results are in agreement with previous experience in this laboratory that even very mild oxidation of the "kerogen" of recent sediments chiefly produces products with short carbon chain length.

\section{DISCUSSION}

The bulk of the organic carbon in this sample consists of high molecular weight, insoluble material that is equivalent to the "kerogen" of ancient marine sediments or to the "humus" fraction of terrestrial soils. Such polymers are difficult to characterize. Degradation by oxidation or by pyrolysis mainly yields small molecules that are difficult to relate to the structure of the material. Higher molecular weight substances, with possibly more structural significance, are produced in very low yield.

The benzene-methanol extract of the original sediment contains only a small amount of the organic carbon but has an abundant amount of "biological marker" molecules which contain some record of the depositional history and subsequent diagenesis. The fatty alcohols and acids with greater than twenty carbon atoms are of interest. They are generally considered to be characteristic of the waxes of higher terrestrial plants and are not believed to be abundant in marine organisms. The dihydrophytol could arise from the phytol side chain of chlorophyl. The porphyrins produced during pyrolysis indicate that chlorophyl derivatives are present. The iso- and anteiso-structures are believed to have arisen from bacteria living in the sediment. The large amount of triterpenoid structures may show a contribution from higher plants.

Sections of this core taken from near the top and the bottom are being studied by the same techniques as described for this sample near the middle. 


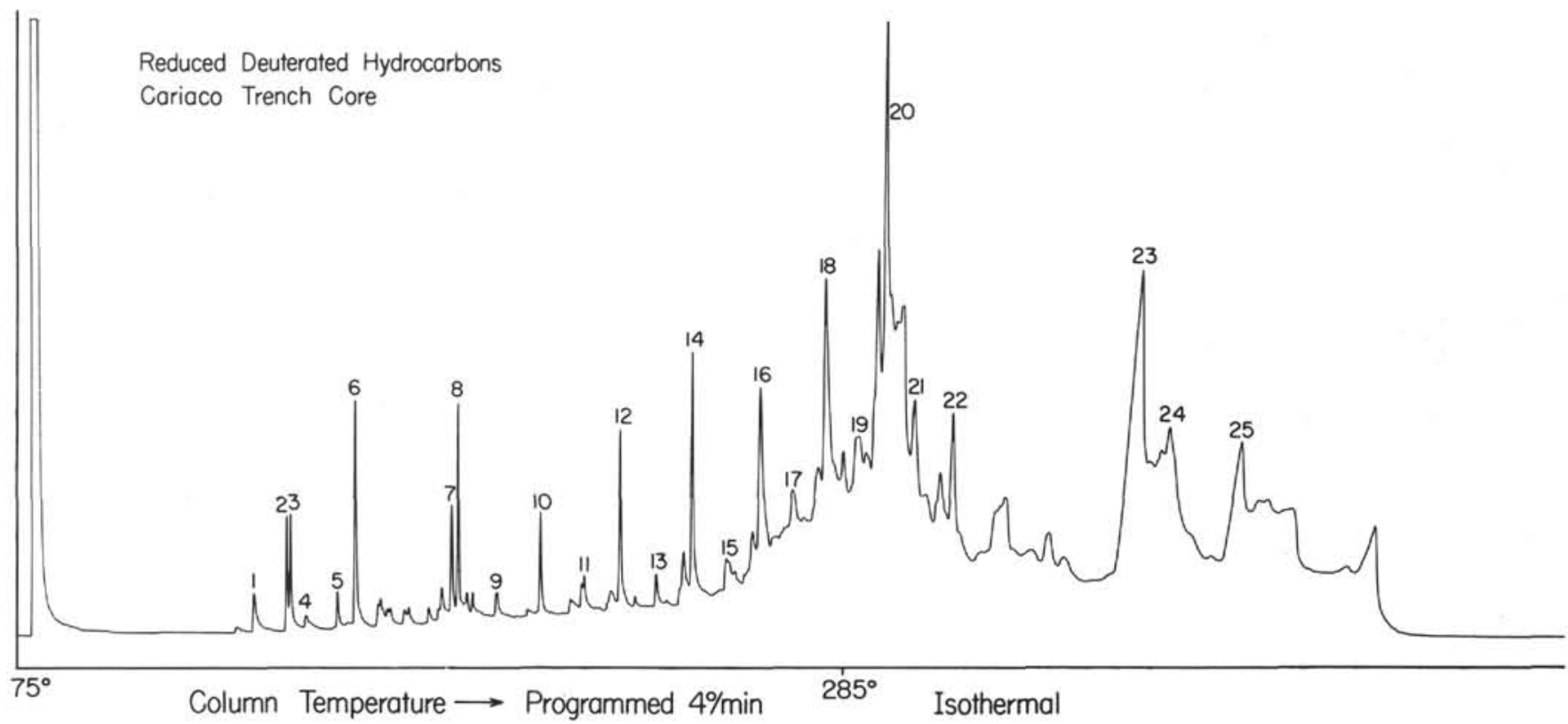

Figure 1. Gas chromotogram of reduced deuterated hydrocarbons - The chromatogram was made on a Perkin-Elmer 900 Gas Chromatograph fitted with a 100 foot by 0.010 inch capillary column coated with $0 \mathrm{~V}-101$ silicone stationary phase. The column head pressure was 30 pounds per square inch and the column temperature was programmed linearly from 75 to $285^{\circ} \mathrm{C}$. at a rate of 4 degree per minute and then held at $285^{\circ}$ for 30 minutes. The identification of the numbered peaks is as follows; (1) n-Tetradecane, (2) Anteiso-pentadecane (3) Iso-pentadecane (4) n-Pentadecane (5) Iso-hexadecane (6) n-Hexadecane (7) n-Octadecane (8) Phytane (9) n-Nonadecane (10)n-Eicosane (11)n-Henicosane (12) n-Docosane (13) n-Tricosane (14)n-Tetracosane (15) n-Pentacosane (16) n-Hexacosane (17)n-Heptacosane (18)n-Octacosane (19) n-Nonacosane (20) n-Triacontane (21)n-Hentriacontane (22) n-Dotriacontane (23) Triterpane hydrocarbons (24) Triterpane hydrocarbons (25) Triterpane hydrocarbons

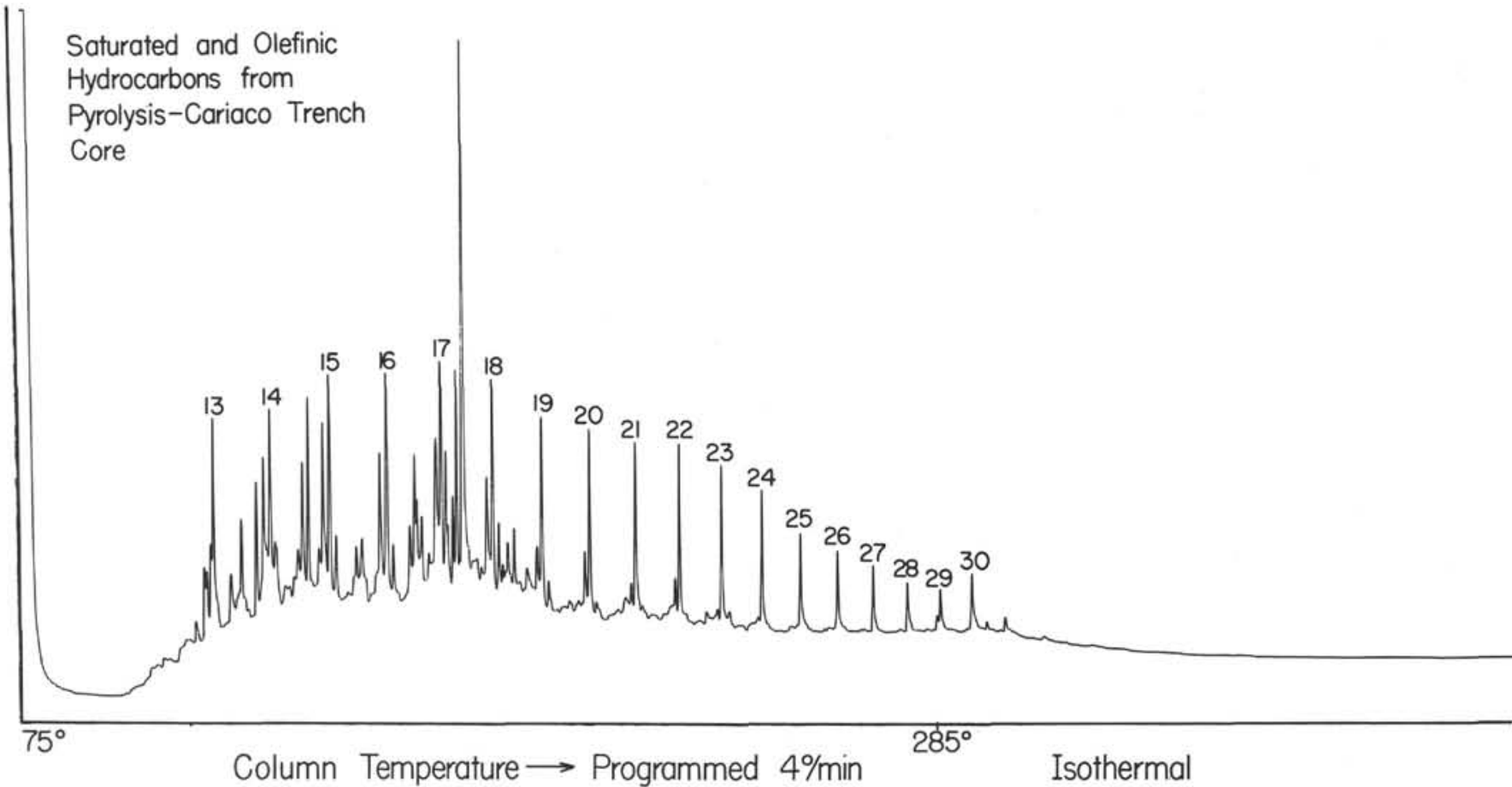

Figure 2. Paraffinic and Olefinic Hydrocarbons from pyrolysis - The gas chromatogram was made in the same way as in Figure 1. The peaks with numbers at their tops are due to normal saturated hydrocarbons with carbon numbers as indicated. The smaller peak immediately preceeding each one is due to the corresponding normal olefin. The largest peak in the chromatogram, lying between C-18 and C-19 is due to an alkyl substituted three ring hydrocarbon. 